\title{
The implication of circulating long non-coding RNA MALAT1 in diagnosis, disease surveillance and prognosis of acute ischemic stroke
}

Hongbo Ren ( $\nabla$ ranxiongqinxz@163.com )

HanDan Central Hospital https://orcid.org/0000-0002-2347-1628

Feng Wu

HanDan Central Hospital

Bin Liu

HanDan Central Hospital

Zhi-Yuan Song

HanDan Central Hospital

Da-Cheng Qu

Hebei University of Engineering

Research article

Keywords: acute ischemic stroke; IncRNA MALAT1; inflammation; survival

Posted Date: September 17th, 2019

DOI: https://doi.org/10.21203/rs.2.14481/v1

License: (9) This work is licensed under a Creative Commons Attribution 4.0 International License.

Read Full License 


\section{Abstract}

Background We aimed to investigate predictive value of long non-coding RNA metastasis-associated lung adenocarcinoma transcript 1 (Inc-MALAT1) for acute ischemic stroke (AIS) risk, and the association of Inc-MALAT1 expression with disease severity, inflammation as well as recurrence free survival (RFS) in AIS patients.

Methods 120 AIS patients and 120 controls were recruited. Venous blood samples from AIS patients (within 24 hours after symptoms onset) and controls (at entry to study) were collected to detect plasma Inc-MALAT1 expression by real-time quantitative polymerase chain reaction. For AIS patients, AIS severity was assessed by NIHSS score; plasma concentrations of inflammation factors (including C-reactive protein (CRP), tumor necrosis factor a (TNF-a), interleukin (IL)-6, IL-8, IL-10, IL-17 and IL-22) were measure; and RFS was calculated.

Results Lnc-MALAT1 expression was decreased in AIS patients compared to controls, and it had a good predictive value for AIS risk (AUC=0.791, 95\% Cl: 0.735-0.846). For disease condition, Inc-MALAT1 expression negatively correlated with NIHSS score and pro-inflammatory factors expressions (including CRP, TNF-a, IL-6, IL-8 and IL-22), while it positively correlated with anti-inflammatory factor IL-10 expression. Besides, Inc-MALAT1 expression was elevated in AIS complicated with diabetes but numerically reduced in AIS complicated with hepertension. For prognosis, Inc-MALAT1 high expression numerically correlated with longer RFS, but without statistical significance.

Conclusion Inc-MALAT1 is downregulated and has a good predictive value of AIS risk, and its high expression correlates with decreased NIHSS score, reduced inflammation, as well as numerically better RFS in AIS patients.

\section{Background}

Acute ischemic stroke (AIS), a common disorder caused by embolic or thromboembolic occlusion in an artery supplying the brain, results in irreversible infarction of brain tissue and functional impairment, which brings economic and social burden for families.[1-3] Current treatments of AIS mainly focus on recovering cerebral circulation and lead to a great likelihood of independent living following AIS, while several restrictions exist in current therapy (such as narrow treatment time window and low recanalization rate), and the prognosis of AIS is still far from satisfactory.[4-6] Increasing studies disclose that early identification of AIS risk and prediction of prognosis may help improve treatment outcomes of AIS patients. $[7,8]$ Thus, searching for convincing biomarkers that monitoring AIS risk and predicting prognosis is urgently needed.

Long non-coding RNAs (IncRNAs) are transcripts with more than 200 nucleotides and rarely encode proteins, which play essential roles in regulation of protein-coding genes and signaling pathways related to development of diseases.[8] LncRNA metastasis-associated lung adenocarcinoma transcript 1 (IncMALAT1), located on human chromosome 11q13.1, is a IncRNA abundantly expressed and evolutionarily 
conserved throughout a variety of mammalian species.[7] Accumulating data from basic and clinical studies have disclosed that Inc-MALAT1 could protect ischemia-induced brain microvascular endothelial cells (BMECs), implying that Inc-MALAT1 might regulate cerebrovascular pathologies in stroke. $[9,10]$ Meanwhile, Inc-MALAT1 has been reported to be downregulated and primarily plays an anti-inflammatory role in neurological diseases patients as well as cardiovascular and cerebrovascular diseases patients. $[11,12]$ Considering the participation of Inc-MALAT1 in cerebrovascular pathologies of stroke and its antiinflammation effect on cardiovascular and cerebrovascular diseases, we hypothesized that Inc-MALAT1 might serve as a biomarker for disease risk and progression in AIS through affecting inflammation level, whereas related evidences were seldomly reported. Hence, we conducted this study to investigate the predictive value of Inc-MALAT1 for AIS risk, and the association of Inc-MALAT1 expression with disease severity, inflammation level as well as recurrence free survival (RFS) in AIS patients.

\section{Methods}

\section{Participants}

In total, 120 patients with AIS and 120 controls with high stroke risk factors were recruited from our hospital between July 2014 and June 2017. For the AIS patients, screening criteria were as follows: (i) primary diagnosis of AIS by clinical presentation, brain non-contrast computed tomography (CT) or diffusion-weighted magnetic resonance imaging, according to the "Guidelines for the Early Management of Patients with Acute Ischemic Stroke: A Guideline for Healthcare Professionals from the American Heart Association/American Stroke Association (AHA/ASA)"[13]; (ii) age more than 18 years; (iii) admitted to our hospital within 24 hours after the symptoms onset; (iv) able to be regularly followed up. Following AIS patients were excluded: (i) had evidence of intracranial hemorrhage; (ii) treated with immunosuppressant within 1 month; (iii) suffered from infection (active or in the preceding 14 days of stroke); (iv) accompanied with hematological malignancies or solid tumors; $(v)$ women who were pregnant or nursing. As for the controls, all of them had no history of stroke or malignancies and were presented with at least three stroke risk factors, such as hypertension, diabetes mellitus, hyperlipidemia, smoke and so on. The Institutional Review Board of our hospital approved the study protocol, and all participants or their guardians provided written informed consents.

\section{Data collection and stroke severity assessment}

At entry to the study, the baseline data of AIS patients was obtained through interview and medical records, which included age, gender, body mass index (BMI), smoke, hypertension, diabetes mellitus, hyperlipidemia, hypeluricemia and chronic kidney disease (CKD). Severity of AIS was assessed within the day of admission by use of the National Institutes of Health Stroke Scale (NIHSS) score. The NIHSS score aimed at assessing neurological impairment ranging from 0 to 42, a higher score indicated a more serious nerve damage, and the classification of severity was as follows: 0-1 point, normal or near normal; 2-4 points, mild stroke; 5-15 points, moderate stroke; $16-20$ points, moderate-severe stroke; $21-42$ points, 
severe stroke. Besides, controls' basic characteristics including age, gender, BMI, smoke, hypertension, diabetes mellitus, hyperlipidemia, hypeluricemia and CKD were also documented on the enrollment.

\section{Blood samples collection and determination}

Venous blood samples were collected from AIS patients (within 24 hours after symptoms onset) and controls using ethylene diamine tetraacetic acid (EDTA) tubes, and subsequently centrifuged at $1600 \mathrm{~g}$ for $10 \mathrm{~min}$ (within $30 \mathrm{~min}$ ) to acquire supernatant, then the supernatant was further centrifuged at $16000 \mathrm{~g}$ for $10 \mathrm{~min}$ to obtain the plasma, which was scored at $-80^{\circ} \mathrm{C}$ for further analysis. Lnc-MALAT1 relative expression in the plasma of AIS patients and controls was determined by real-time quantitative polymerase chain reaction (RT-qPCR). AIS patients' CRP concentration in plasma was detected using Fully automatic POCT fluorescence immunoassay analyzer (GeteinBiotech, Nanjing, Jiangsu, China), and the plasma levels of inflammatory cytokines including tumor necrosis factor a (TNF-a), interleukin-6 (IL6), IL-8, IL-10, IL-17 and IL-22 were measured by human enzyme-linked immunosorbent assay (ELISA) kits (Thermo Fisher Scientific, Waltham, Massachusetts, USA) according to the manufacturer's recommendations.

\section{RT-qPCR}

Using QIAamp RNA Blood Mini Kit (Qiagen, Duesseldorf, Nordrhein-Westfalen, German), total RNA was extracted from plasm samples. Then, reverse transcription to cDNA was performed by PrimeScript ${ }^{T M} R T$ reagent Kit (Perfect Real Time) (Takara, Dalian, Liaoning, China), and qPCR was performed using TB Green $^{\text {TM }}$ Fast qPCR Mix (Takara, Dalian, Liaoning, China)). GAPDH was applied as the internal reference. Primers used in the RT-qPCR were as follows: Inc-MALAT1, forward ( $\left.5^{\prime}->3^{\prime}\right)$ :

TCCTAAGGTCAAGAGAAGTGTCAG, reverse (5'->3'): GTGGCGATGTGGCAGAGAA; GAPDH, forward (5'->3'): TGACCACAGTCCATGCCATCAC, reverse (5'->3'): GCCTGCTTCACCACCTTCTTGA.

\section{Follow up}

All AIS patients received appropriate treatments as recommended by 2013 AlS Guidelines (AHA/ASA), and were followed up regularly or as clinically indicated. The last follow-up date was June 30, 2018, and the median follow-up duration was 25.0 months (range: 1.0-42.0 months). RFS was calculated from the date of hospital admission to the date of recurrence or death.

\section{Statistical analysis}

SPSS 24.0 statistical software (SPSS Inc, Chicago, IL, USA) was used for statistical data processing, and the GraphPad Prism 6.01 (GraphPad Software Inc., San Diego, CA, USA) was applied to the graphs plotting. Continuous variables were expressed as mean \pm standard deviation (SD) or median and interquartile range (IQR), and the categorical variables were expressed as number (percentage). Differences between groups were determined by Student's $t$ test, Wilcoxon rank sum test or Chi-square test. Correlations between variables were analyzed by Spearman rank test. Diagnostic value of variable was assessed by receiver operating characteristic (ROC) curve analysis and the derived area under the 
curve (AUC) as well as 95\% confidence interval (Cl). RFS profiles were illuminated by plotting the KaplanMeier (K-M) curve, and the difference of RFS between groups was determined by the log-rank test. Factors affecting RFS were analyzed by univariate and multivariate Cox's proportional hazards regression models. All tests were 2 -sided, and $P$ values $<0.05$ were considered statistically significant.

\section{Results}

\section{Characteristics in AIS patients and controls}

120 AIS patients and 120 controls were enrolled in this study (Table 1). The mean age of AIS patients and controls were $62.4 \pm 12.3$ years and $61.1 \pm 11.0$ years respectively. Meanwhile, there were 82 males as well as 38 females in AlS patients, and 87 males as well as 33 females in controls. Besides, mean BMI was $24.6 \pm 2.8 \mathrm{~kg} / \mathrm{m}^{2}$ and $24.1 \pm 2.8 \mathrm{~kg} / \mathrm{m}^{2}$ in AIS patients and controls respectively. No difference of age $(P=$ $0.363)$, gender $(P=0.479), \mathrm{BMI}(P=0.159)$ between AIS patients and controls. Also, the percentage of smoke $(P=0.796)$, hypertension $(P=0.421)$, diabetes mellitus $(P=0.182)$, hyperlipidemia $(P=0.296)$, hypeluricemia $(P=0.888)$ as well as $\operatorname{CKD}(P=0.360)$ was similar between two groups. The detailed information about other baseline characteristics were shown in Table 1.

\section{Disease severity and inflammatory factors in AIS patients}

In AIS patients, mean NIHSS score was $7.8 \pm 3.5$ and the median NIHSS score was 7.0 (5.0-10.0) (Table 2). As to inflammatory factors, mean values of CRP, TNF-a, IL-6, IL-8, IL-10, IL-17 and IL-22 were $35.3 \pm$ $21.6 \mathrm{mg} / \mathrm{L}, 79.5 \pm 56.1 \mathrm{pg} / \mathrm{mL}, 51.7 \pm 29.9 \mathrm{pg} / \mathrm{mL}, 62.0 \pm 35.1 \mathrm{pg} / \mathrm{mL}, 23.3 \pm 12.6 \mathrm{pg} / \mathrm{mL}, 101.9 \pm 49.1$ $\mathrm{pg} / \mathrm{mL}$ and $85.8 \pm 64.9 \mathrm{pg} / \mathrm{mL}$, and the median values of these factors were $29.0(23.2-38.7) \mathrm{mg} / \mathrm{L}, 60.1$ (44.9-94.6) pg/mL, 44.7 (38.1-54.3) pg/mL, 50.4 (40.2-76.4) pg/mL, 20.9 (14.1-30.9) pg/mL, 96.2 (65.3122.7) $\mathrm{pg} / \mathrm{mL}$ and $67.8(48.5-96.9) \mathrm{pg} / \mathrm{mL}$ respectively.

\section{Comparison of Inc-MALAT1 expression between AIS patients and controls}

Lnc-MALAT1 expression was decreased in AIS patients compared to controls $(P<0.001)$ (Figure 1A). And ROC curve showed that Inc-MALAT1 had a good predictive value for AIS risk (AUC=0.791, 95\% Cl: 0.7350.846) (Figure 1B).

\section{Correlation of Inc-MALAT1 expression with NIHSS score in AIS patients}

Spearman rank test was used to determine the correlation of Inc-MALAT1 expression with NIHSS score, which showed that Inc-MALAT1 expression was negatively correlated with NIHSS score in AIS patients ( $P$ $<0.001, r=-0.437$ ) (Figure 2).

\section{Correlation of Inc-MALAT1 expression with inflammatory factors in AIS patients}

Lnc-MALAT1 expression was negatively associated with expressions of inflammatory factors including CRP $(P<0.001, r=-0.354)$ (Figure 3A), TNF-a $(P=0.021, r=-0.211)$ (Figure 3B), IL-6 $(P<0.001, r=0.328)$ 
(Figure 3C), IL-8 ( $P=0.037, \mathrm{r}=-0.191)$ (Figure 3D) and IL-22 ( $P=0.017, \mathrm{r}=-0.218)$ (Figure 3G), while it was positively correlated with expression of anti-inflammatory cytokine IL-10 $(P=0.010, r=0.235)$ (Figure 3E) in AIS patients. Besides, no correlation of Inc-MALAT1 expression with IL-17 expression ( $P=$ $0.250, r=-0.106$ ) (Figure 3F) was found.

\section{Correlation of Inc-MALAT1 expression with complications in AIS patients}

To investigate the correlation of Inc-MALAT1 expression with complications in AIS patients, all patients were categorized into different subgroups according to their complications (Figure 4). Lnc-MALAT1 expression was elevated in diabetes compared to non-diabetes $(P=0.017)$ (Figure 4B), whereas it was numerically reduced in hypertension patients compared with non-hypertension patients $(P=0.081)$ (Figure 4A). Besides, no difference of Inc-MALAT1 expression was found between hyperlipidemia patients and non-hyperlipidemia patients $(P=0.391)$ (Figure $4 C)$, hypeluricemia patients and non-hypeluricemia patients $(P=0.657)$ (Figure 4D), or CKD patients and non-CKD patients $(P=0.264)$ (Figure 4E). These data indicated that Inc-MALAT1 expression was positively correlated with diabetes occurrence in AIS patients.

\section{Comparison of RFS between Inc-MALAT1 high expression patients and Inc-MALAT1 low expression patients}

Patients were divided into Inc-MALAT1 high expression group and Inc-MALAT1 low expression group according to the median value of Inc-MALAT1 expression. RFS in AIS patients with Inc-MALAT1 high expression was numerically longer compared to AIS patients with Inc-MALAT1 low expression, but without statistical significance $(P=0.053)$ (Figure 5).

\section{Analysis of factors affecting RFS in AlS patients}

Univariate Cox's regression analysis displayed that Inc-MALAT1 high expression was numerically associated with better RFS in AIS patients (although no statistical significance) $(P=0.063)$, while CRP ( $\geq 29.0$ vs. $<29.0 \mathrm{mg} / \mathrm{L}$ ) was associated with worse RFS $(P=0.006)$ (Table 3$)$. Moreover, multivariate Cox's regression analysis showed that Inc-MALAT1 high expression was not an independent predictive factor for RFS, whereas CRP ( $\geq 29.0$ vs. $<29.0 \mathrm{mg} / \mathrm{L}$ ) was an independent factor predicting reduced RFS $(P=0.024)$.

\section{Discussion}

Our results indicated that: (1) Inc-MALAT1 expression was decreased in AIS patients compared to controls, and it had a good predictive value of AIS risk; (2) Inc-MALAT1 high expression correlated with decreased NIHSS score as well as reduced inflammatory factors levels in AIS patients; (3) Inc-MALAT1 high expression numerically correlated with prolonged RFS in AIS patients, but without statistical significance. 
LncRNAs are involved in a variety of biological processes (such as genes transcription, organizing RNAprotein complex, and management in protein activity).[14-17] As one of the frequently investigated IncRNAs, Inc-MALAT1 has various molecular functions (including alternative splicing, transcriptional regulation and post-transcriptional regulation), and also participates in multiple physiological functions (including neural development, skeletal myogenesis and vascular growth).[7] As to its role in neurological diseases or cerebrovascular diseases, previous studies demonstrate that Inc-MALAT1 has protective effects in these diseases via repressing proapoptotic or pro-inflammatory factors.[10,11,18, 19] For instance, a study displays that Inc-MALAT1 protects human brain vascular endothelial cells from OGDinduced apoptosis through activating phosphatidylinositol 3-kinase (PI3K).[19] In addition, Inc-MALAT1 expression is decreased in the spinal cords of mice with experimental autoimmune encephalomyelitis, and its knockdown raises levels of inflammatory cytokines (including IL-1 and IL-6).[11] Also, silencing of Inc-MALAT1 raises the levels of proapoptotic factor Bim and pro-inflammatory cytokines (including MCP1 as well as E-selectin) in BMECs following oxygen-glucose deprivation (OGD), which is an in vitro mimic of ischemic stroke conditions.[10] Another study discloses that Inc-MALAT1 knockdown aggravates OGDinduced overexpression of pro-inflammatory cytokines including MCP-1 and IL-6 in mouse cerebral microvascular endothelial cells.[18] These studies reveal that Inc-MALAT1 has protective effects in neurological diseases or cerebrovascular diseases through decreasing inflammation level or inhibiting cell apoptosis.

Previous clinical trials mainly focus on the exploration of Inc-MALAT1 in cancer patients, which display that Inc-MALAT1 plays a tumor-promotive role in these cancer patients, while the information about the role of Inc-MALAT1 in neurological diseases patients or cerebrovascular diseases patients is still limited. [20-22] Just a few studies display that Inc-MALAT1 expression is reduced in central nervous system tissues from multiple sclerosis patients and carotid plaques from atherosclerosis patients.[11, 12] As for the correlation of Inc-MALAT1 with disease severity and inflammation in neurological diseases or cerebrovascular diseases patients, only a study shows that elevated Inc-MALAT1 expression is correlated with less advanced lesions in atherosclerosis patients.[12] Considering that Inc-MALAT1 might participate in the cerebrovascular pathologies of stroke according to previous studies, meanwhile, it presented antiinflammatory effect in cardiovascular and cerebrovascular diseases, we hypothesized that Inc-MALAT1 expression might be related to the disease risk, progression or inflammation level of AIS, while related explorations in AIS are seldomly reported.[9-12, 23] In our study, we assessed the predictive value of IncMALAT1 for AIS risk, and we discovered that Inc-MALAT1 expression was lower in AIS patients compared to controls, and Inc-MALAT1 expression presented good diagnostic value for AIS, which might due to its protective effect in these diseases via repressing cell apoptosis of BMECs. Moreover, we investigated the correlation of Inc-MALAT1 expression with disease severity as well as inflammation in AIS patients, and we observed that Inc-MALAT1 high expression was associated with decreased NIHSS score, reduced levels of inflammatory factors (including CRP, TNF-a, IL-6, IL-8 and IL-22), meanwhile it reduced proportion of diabetes but numerically increased proportion of hypertension (without significant difference) in AIS patients. The possible reasons of these results might be as follows: (1) Inc-MALAT1 had protective influence on brain microvascular endothelial cells through inhibiting cell apoptosis, thereby 
contributed to maintaining a healthy brain endothelium that was essential for normal cerebrovascular physiology, therefore the severity of AIS was attenuated and decreased NIHSS score was observed[7, 18]; (2) Inc-MALAT1 might decrease the expression of transcriptional factor nuclear factor-kappa B (NF-kB) that drove the transcription of a series of inflammatory factors, thus led to reduced inflammatory factors levels and alleviative inflammation in AIS patients[24]; (3) Inc-MALAT1 protected the brain microvascular endothelial cells against apoptosis, thereby facilitated microvascular permeability and helped restored cerebral vasoreactivity, thus it might attenuate hypertension in AIS patients[23] (4) Inc-MALAT1 was found to interact with transcription factor Foxo1 and SIRT1 transcription to induce poor glycemic control and insulin resistance, which might promote the occurrence of diabetes.[25, 26]

As to the prognostic value of Inc-MALAT1 in human diseases, it has been identified as a valuable biomarker for disease prognosis in several cancers, while limited clinical studies have been found in AIS, only a study displays that Inc-MALAT1 high expression is associated with prolonged main adverse cardiovascular and cerebrovascular events (MACCE)-free survival in atherosclerosis patients.[12] To our knowledge, there was still no evidence about the influence of Inc-MALAT1 on the prognosis in AIS patients. In our study, we observed that Inc-MALAT1 expression was numerically positively correlated with RFS in AIS patients, but without significant difference. These results might be due to: (1) Inc-MALAT1 reduced the production of inflammation cytokines and further decreased inflammation and attenuated disease progression, thus led to better RFS in AIS patients[10, 18]; (2) Inc-MALAT1 might facilitate sensitivity to treatment in AIS, thereby increased treatment efficacy and resulted in longer RFS in AIS patients.

Some limitations existed in our study: (1) sample size of 120 AIS patients was relatively small, thus the statistical power might be low; (2) the median follow-up duration (25.0 months (range: 1.0-42.0 months)) was relatively short, thus the correlation of Inc-MALAT1 expression with long-term prognosis was not investigated; (3) detailed mechanism of Inc-MALAT1 in AIS was still unclear, and further study was still needed.

\section{Conclusions}

In conclusion, Inc-MALAT1 is downregulated and has a good predictive value of AIS risk, and its high expression correlates with decreased NIHSS score, reduced inflammation, as well as numerically better RFS in AIS patients.

\section{Abbreviations}

Inc-MALAT1: long non-coding RNA metastasis-associated lung adenocarcinoma transcript 1; AIS: acute ischemic stroke; RFS: recurrence free survival; CRP: C-reactive protein; TNF-a: tumor necrosis factor a; IncRNAs: Long non-coding RNAs; BMECs: brain microvascular endothelial cells; CT: computed tomography; BMI: body mass index; CKD: chronic kidney disease; EDTA: ethylene diamine tetraacetic acid; RT-qPCR: real-time quantitative polymerase chain reaction 


\section{Declarations}

\section{Acknowledgments}

Not applicable.

\section{Ethics approval and written consent to participate}

The Institutional Review Board of HanDan Central Hospital approved the study protocol, and all participants or their guardians provided written informed consents.

\section{Authors contributions}

HR designed and approved the entire study; HR prepared the draft; FW, BL and ZS collected and analyzed the data; DQ revised the manuscript; All authors have read and approved the final manuscript.

\section{Funding}

None.

\section{Availability of data and materials}

The datasets used and/or analyzed during the current study are available from the corresponding author on reasonable request.

\section{Consent for publication}

Not applicable.

\section{Competing interests}

The authors declare that they have no competing interests.

\section{References}

1. Li Y, Zhong W, Jiang Z, Tang X. New progress in the approaches for blood-brain barrier protection in acute ischemic stroke. Brain research bulletin. 2019;144:46-57.

2. Heit JJ, Zaharchuk G, Wintermark M. Advanced Neuroimaging of Acute Ischemic Stroke: Penumbra and Collateral Assessment. Neuroimaging clinics of North America. 2018;28 4:585-97.

3. Wu C, Wu D, Chen J, Li C, Ji X. Why not Intravenous Thrombolysis in Patients with Recurrent Stroke within 3 Months? Aging and disease. 2018;9 2:309-16.

4. Luo Y, Tang H, Li H, Zhao R, Huang Q, Liu J. Recent advances in the development of neuroprotective agents and therapeutic targets in the treatment of cerebral ischemia. European journal of medicinal chemistry. 2019;162:132-46. 
5. Sun MS, Jin H, Sun X, Huang S, Zhang FL, Guo ZN, et al. Free Radical Damage in IschemiaReperfusion Injury: An Obstacle in Acute Ischemic Stroke after Revascularization Therapy. Oxidative medicine and cellular longevity. 2018;2018:3804979.

6. Leiva-Salinas C, Patrie JT, Xin W, Michel P, Jovin T, Wintermark M. Prediction of Early Arterial Recanalization and Tissue Fate in the Selection of Patients With the Greatest Potential to Benefit From Intravenous Tissue-Type Plasminogen Activator. Stroke. 2016;47 2:397-403.

7. Zhang $\mathrm{X}$, Hamblin MH, Yin KJ. The long noncoding RNA Malat1: Its physiological and pathophysiological functions. RNA Biol. 2017;14 12:1705-14.

8. Ren W, Yang X. Pathophysiology of Long Non-coding RNAs in Ischemic Stroke. Frontiers in molecular neuroscience. 2018;11:96.

9. Yang H, Xi X, Zhao B, Su Z, Wang Z. KLF4 protects brain microvascular endothelial cells from ischemic stroke induced apoptosis by transcriptionally activating MALAT1. Biochemical and biophysical research communications. 2018;495 3:2376-82.

10. Zhang X, Tang X, Liu K, Hamblin MH, Yin KJ. Long Noncoding RNA Malat1 Regulates Cerebrovascular Pathologies in Ischemic Stroke. The Journal of neuroscience : the official journal of the Society for Neuroscience. 2017;37 7:1797-806.

11. Masoumi F, Ghorbani S, Talebi F, Branton WG, Rajaei S, Power C, et al. Malat1 long noncoding RNA regulates inflammation and leukocyte differentiation in experimental autoimmune encephalomyelitis. Journal of neuroimmunology. 2019;328:50-9.

12. Cremer S, Michalik KM, Fischer A, Pfisterer L, Jae N, Winter C, et al. Hematopoietic Deficiency of the Long Noncoding RNA MALAT1 Promotes Atherosclerosis and Plaque Inflammation. Circulation. 2019;139 10:1320-34.

13. Jauch EC, Saver JL, Adams HP, Jr., Bruno A, Connors JJ, Demaerschalk BM, et al. Guidelines for the early management of patients with acute ischemic stroke: a guideline for healthcare professionals from the American Heart Association/American Stroke Association. Stroke. 2013;44 3:870-947.

14. Chen $H$, Wang $X$, Yan X, Cheng X, He X, Zheng W. LncRNA MALAT1 regulates sepsis-induced cardiac inflammation and dysfunction via interaction with miR-125b and p38 MAPK/NFkappaB. International immunopharmacology. 2018;55:69-76.

15. Dai L, Zhang G, Cheng Z, Wang X, Jia L, Jing X, et al. Knockdown of LncRNA MALAT1 contributes to the suppression of inflammatory responses by up-regulating miR-146a in LPS-induced acute lung injury. Connective tissue research. 2018;59 6:581-92.

16. Yu Z, Rayile A, Zhang X, Li Y, Zhao Q. Ulinastatin protects against lipopolysaccharide-induced cardiac microvascular endothelial cell dysfunction via downregulation of IncRNA MALAT1 and EZH2 in sepsis. International journal of molecular medicine. 2017;39 5:1269-76.

17. Brock M, Schuoler C, Leuenberger C, Buhlmann C, Haider TJ, Vogel J, et al. Analysis of hypoxiainduced noncoding RNAs reveals metastasis-associated lung adenocarcinoma transcript 1 as an important regulator of vascular smooth muscle cell proliferation. Experimental biology and medicine. 2017;242 5:487-96. 
18. Zhang J, Yuan L, Zhang X, Hamblin MH, Zhu T, Meng F, et al. Altered long non-coding RNA transcriptomic profiles in brain microvascular endothelium after cerebral ischemia. Experimental neurology. 2016;277:162-70.

19. Xin JW, Jiang YG. Long noncoding RNA MALAT1 inhibits apoptosis induced by oxygen-glucose deprivation and reoxygenation in human brain microvascular endothelial cells. Experimental and therapeutic medicine. 2017;13 4:1225-34.

20. Arun G, Spector DL. MALAT1 long non-coding RNA and breast cancer. RNA Biol. 2019:1-4.

21. Zhuang M, Zhao S, Jiang Z, Wang S, Sun P, Quan J, et al. MALAT1 sponges miR-106b-5p to promote the invasion and metastasis of colorectal cancer via SLAIN2 enhanced microtubules mobility. EBioMedicine. 2019;41:286-98.

22. Li Z, Xu L, Liu Y, Fu S, Tu J, Hu Y, et al. LncRNA MALAT1 promotes relapse of breast cancer patients with postoperative fever. American journal of translational research. 2018;10 10:3186-97.

23. Vitt JR, Trillanes M, Hemphill JC, 3rd. Management of Blood Pressure During and After Recanalization Therapy for Acute Ischemic Stroke. Frontiers in neurology. 2019;10:138.

24. Tian $\mathrm{H}$, Wu M, Zhou P, Huang $\mathrm{C}$, Ye $\mathrm{C}$, Wang $\mathrm{L}$. The long non-coding RNA MALAT1 is increased in renal ischemia-reperfusion injury and inhibits hypoxia-induced inflammation. Renal failure. 2018;40 1:527-33.

25. Sathishkumar C, Prabu P, Mohan V, Balasubramanyam M. Linking a role of IncRNAs (long noncoding RNAs) with insulin resistance, accelerated senescence, and inflammation in patients with type 2 diabetes. Human genomics. 2018;12 1:41.

26. Zhou L, Xu DY, Sha WG, Shen L, Lu GY. Long non-coding RNA MALAT1 interacts with transcription factor Foxo1 to regulate SIRT1 transcription in high glucose-induced HK-2cells injury. Biochemical and biophysical research communications. 2018;503 2:849-55.

\section{Tables}

Table 1 Characteristics of AIS patients and controls

\begin{tabular}{lccc}
\hline Parameters & AIS patients $(\mathrm{N}=120)$ & Controls $(\mathrm{N}=120)$ & $P$ value \\
\hline Age, mean \pm SD, years & $62.4 \pm 12.3$ & $61.1 \pm 11.0$ & 0.363 \\
Gender (male/female) & $82 / 38$ & $87 / 33$ & 0.479 \\
BMI, mean \pm SD, kg/m² & $24.6 \pm 2.8$ & $24.1 \pm 2.8$ & 0.159 \\
Smoke, No. (\%) & $58(48.3)$ & $60(50.0)$ & 0.796 \\
Hypertension, No. (\%) & $108(90.0)$ & $104(86.7)$ & 0.421 \\
Diabetes mellitus, No. (\%) & $26(21.7)$ & $18(15.0)$ & 0.182 \\
Hyperlipidemia, No. (\%) & $55(45.8)$ & $47(39.2)$ & 0.296 \\
Hypeluricemia, No. (\%) & $35(29.2)$ & $36(30.0)$ & 0.888 \\
CKD, No. (\%) & $20(16.7)$ & $15(12.5)$ & 0.360 \\
\hline
\end{tabular}


Differences were determined by Student's t test or Chi-square test. $P$ value $<0.05$ was considered as significant. AIS: acute ischemic stroke; SD: standard deviation; BMI: body mass index; CKD: chronic kidney disease;

Table 2 Disease severity, CRP and inflammatory cytokines of AIS patients

\begin{tabular}{lcccc}
\hline Parameters & Mean & SD & Median & IQR \\
\hline NIHSS score & 7.8 & 3.5 & 7.0 & $5.0-10.0$ \\
CRP, mg/L & 35.3 & 21.6 & 29.0 & $23.2-38.7$ \\
TNF- $\alpha, \mathrm{pg} / \mathrm{mL}$ & 79.5 & 56.1 & 60.1 & $44.9-94.6$ \\
IL-6, pg/mL & 51.7 & 29.9 & 44.7 & $38.1-54.3$ \\
IL-8, pg/mL & 62.0 & 35.1 & 50.4 & $40.2-76.4$ \\
IL-10, pg/mL & 23.3 & 12.6 & 20.9 & $14.1-30.9$ \\
IL-17, pg/mL & 101.9 & 49.1 & 96.2 & $65.3-122.7$ \\
IL-22, pg/mL & 85.8 & 64.9 & 67.8 & $48.5-96.9$ \\
\hline
\end{tabular}

SD: standard deviation; IQR: inter-quartile range; NIHSS: National Institutes of Health Stroke Scale; CRP: C-reactive protein; TNF- $\alpha$ : tumor necrosis factor $\alpha$; IL: interleukin.

Table 3 Factors affecting RFS by Cox's proportional hazards regression model analyses 


\begin{tabular}{|c|c|c|c|c|}
\hline \multirow[t]{2}{*}{ Items } & \multicolumn{2}{|c|}{ Univariate Cox's regression } & \multicolumn{2}{|c|}{$\begin{array}{l}\text { Multivariate Cox's } \\
\text { regression }\end{array}$} \\
\hline & $\begin{array}{c}P \\
\text { value }\end{array}$ & HR $(95 \% \mathrm{CI})$ & $\begin{array}{c}P \\
\text { value }\end{array}$ & HR $(95 \% \mathrm{CI})$ \\
\hline lnc-MALAT1 (high vs. low) & 0.062 & $\begin{array}{c}0.402(0.154- \\
1.047)\end{array}$ & 0.200 & $0.482(0.157-1.473)$ \\
\hline Age ( $\geq 61$ vs. $<61$ years) & 0.976 & $\begin{array}{l}0.986(0.406- \\
2.397)\end{array}$ & 0.974 & $0.981(0.307-3.137)$ \\
\hline Gender (male/female) & 0.853 & $\begin{array}{c}1.095(0.421- \\
2.850)\end{array}$ & 0.965 & $0.974(0.304-3.120)$ \\
\hline BMI ( $\geq 24.2$ vs. $<24.2 \mathrm{~kg} / \mathrm{m}^{2}$ ) & 0.506 & $\begin{array}{l}0.741(0.307- \\
1.791)\end{array}$ & 0.725 & $0.835(0.305-2.282)$ \\
\hline Smoke (yes vs. no) & 0.456 & $\begin{array}{l}0.712(0.291- \\
1.741)\end{array}$ & 0.509 & $0.726(0.281-1.876)$ \\
\hline Hypertension (yes vs. no) & 0.836 & $\begin{array}{c}1.167(0.270- \\
5.047)\end{array}$ & 0.704 & $1.371(0.269-6.980)$ \\
\hline Diabetes mellitus (yes vs. no) & 0.193 & $\begin{array}{c}0.378(0.088- \\
1.633)\end{array}$ & 0.526 & $0.585(0.111-3.075)$ \\
\hline Hyperlipidemia (yes vs. no) & 0.853 & $\begin{array}{l}0.920(0.380- \\
2.227)\end{array}$ & 0.978 & $1.015(0.365-2.821)$ \\
\hline Hypeluricemia (yes vs. no) & 0.958 & $\begin{array}{c}1.026(0.394- \\
2.673)\end{array}$ & 0.712 & $0.819(0.283-2.370)$ \\
\hline CKD (yes vs. no) & 0.821 & $\begin{array}{c}1.136(0.377- \\
3.418)\end{array}$ & 0.609 & $1.435(0.360-5.728)$ \\
\hline NIHSS score ( $\geq 7$ vs. $<7$ ) & 0.428 & $\begin{array}{l}0.701(0.292- \\
1.687)\end{array}$ & 0.229 & $0.557(0.215-1.445)$ \\
\hline CRP ( $\geq 29.0$ vs. $<29.0 \mathrm{mg} / \mathrm{L})$ & 0.006 & $\begin{array}{c}4.683(1.563- \\
14.028)\end{array}$ & 0.024 & $\begin{array}{l}4.720(1.224- \\
18.197)\end{array}$ \\
\hline $\begin{array}{l}\text { TNF- } \alpha(\geq 60.1 \text { vs. }<60.1 \\
\mathrm{pg} / \mathrm{mL})\end{array}$ & 0.126 & $\begin{array}{l}2.052(0.818- \\
5.151)\end{array}$ & 0.350 & $1.879(0.501-7.041)$ \\
\hline IL-6 ( $\geq 44.7$ vs. $<44.7 \mathrm{pg} / \mathrm{mL}$ ) & 0.301 & $\begin{array}{c}1.605(0.655- \\
3.931)\end{array}$ & 0.899 & $1.069(0.381-2.996)$ \\
\hline IL-8 ( $\geq 50.4$ vs. $<50.4$ pg/mL) & 0.988 & $\begin{array}{l}0.993(0.413- \\
2.387)\end{array}$ & 0.387 & $0.559(0.149-2.088)$ \\
\hline $\begin{array}{l}\text { IL-10 ( } \geq 20.9 \text { vs. }<20.9 \\
\mathrm{pg} / \mathrm{mL})\end{array}$ & 0.133 & $\begin{array}{l}0.493(0.196- \\
1.239)\end{array}$ & 0.548 & $0.707(0.227-2.195)$ \\
\hline IL-17 ( $\geq 96.2$ vs. 96.2 pg/mL) & 0.586 & $\begin{array}{c}0.783(0.324- \\
1.889)\end{array}$ & 0.153 & $0.474(0.170-1.319)$ \\
\hline $\begin{array}{l}\text { IL-22 ( } \geq 67.8 \text { vs. }<67.8 \\
\mathrm{pg} / \mathrm{mL})\end{array}$ & 0.299 & $\begin{array}{c}1.608(0.656- \\
3.942)\end{array}$ & 0.716 & $0.797(0.235-2.705)$ \\
\hline
\end{tabular}


$P$ value $<0.05$ was considered significant. RFS: recurrence free survival; HR: hazard ratio; CI: confidence interval; BMI: body mass index; CKD: chronic kidney disease; NIHSS:

National Institutes of Health Stroke Scale; CRP: C-reactive protein; TNF- $\alpha$ : tumor necrosis factor $\alpha$; IL: interleukin.

\section{Figures}

A

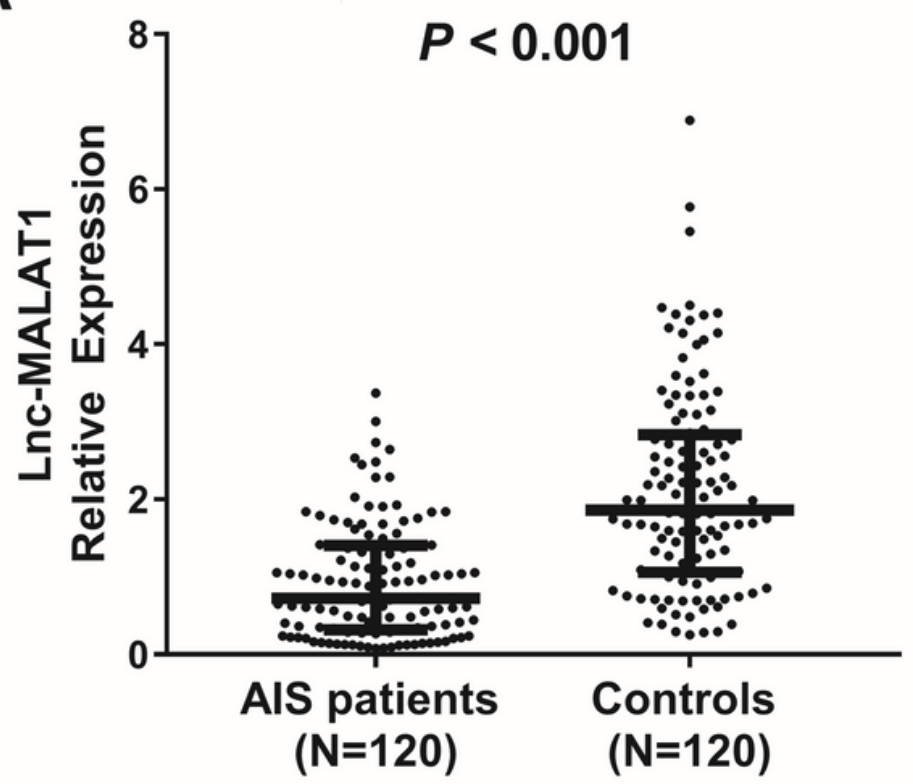

B

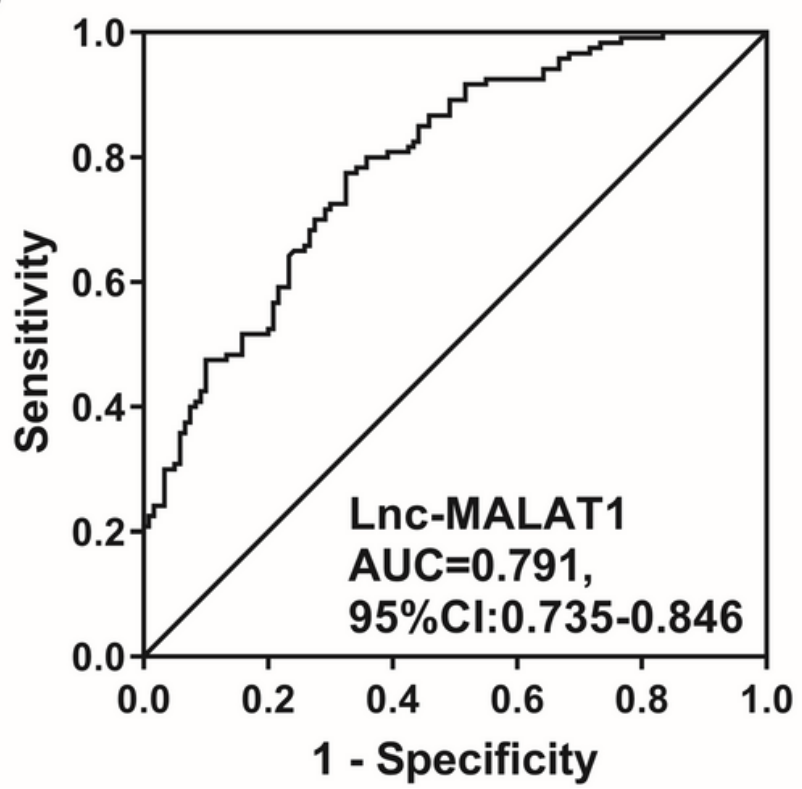

Figure 1

Inc-MALAT1 expression in AIS patients and controls. Lnc-MALAT1 relative expression in AIS patients and controls (A). Diagnostic value of Inc-MALAT1 for AIS assessed by ROC curve (B). Comparison between two groups was determined by Wilcoxon rank sum test. Lnc-MALAT1, long non-coding RNA metastasisassociated lung adenocarcinoma transcript 1; AIS, acute ischemic stroke; ROC curve, receiver operating characteristic curve. $\mathrm{P}<0.05$ was considered significant. 


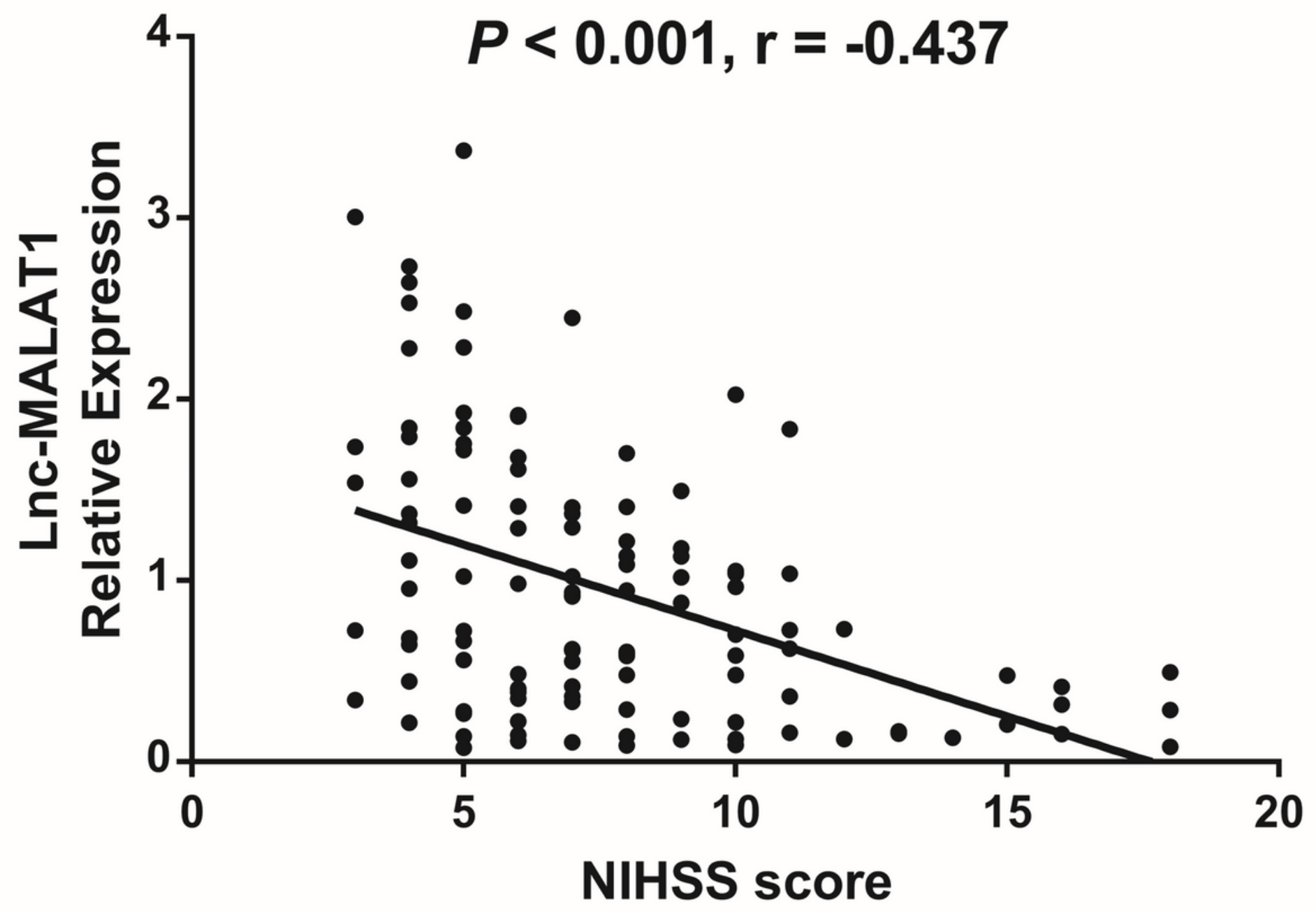

Figure 2

Correlation of Inc-MALAT1 expression with NIHSS score. Lnc-MALAT1 high expression was correlated with decreased NIHSS score. Correlation of Inc-MALAT1 expression with NIHSS score was determined by Spearman rank test. Lnc-MALAT1, long non-coding RNA metastasis-associated lung adenocarcinoma transcript 1; NIHSS score, National Institutes of Health Stroke Scale score. $P<0.05$ was considered significant. 
A

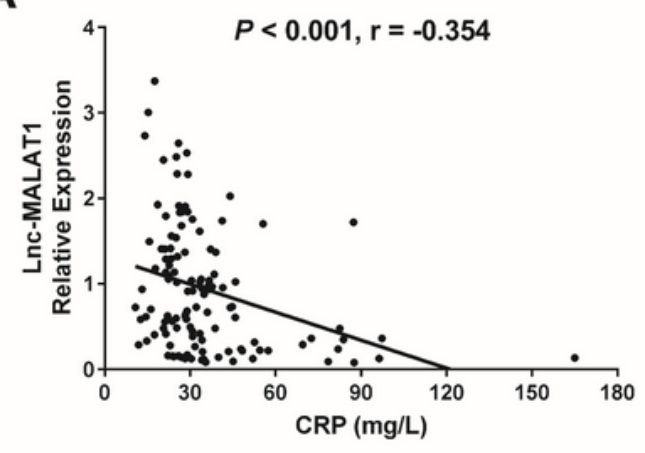

C

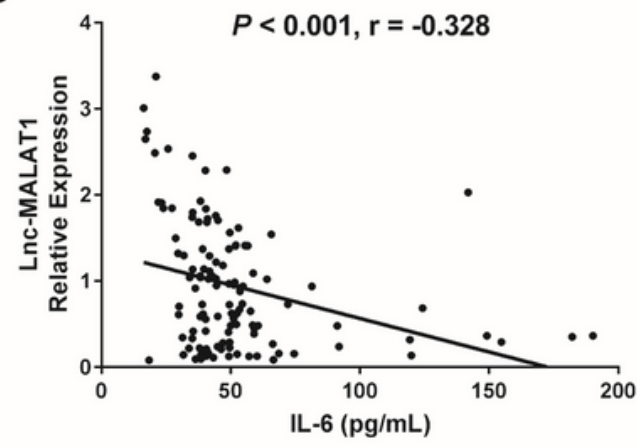

E

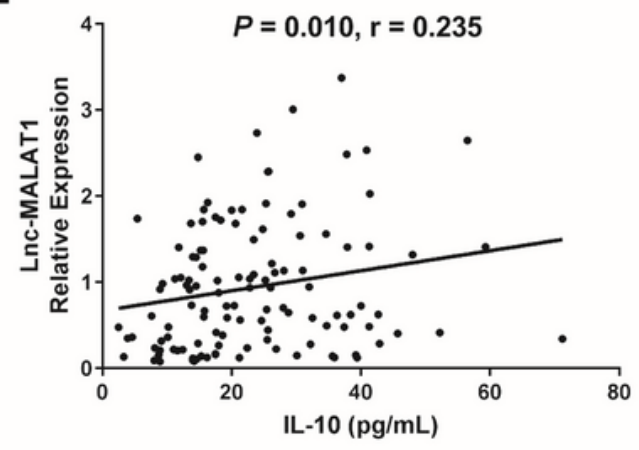

G

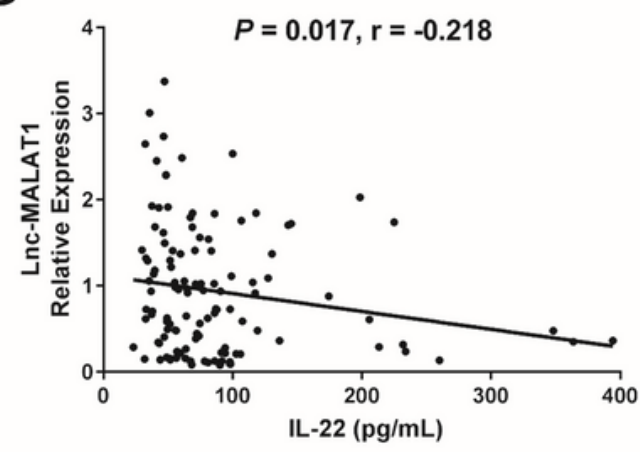

B

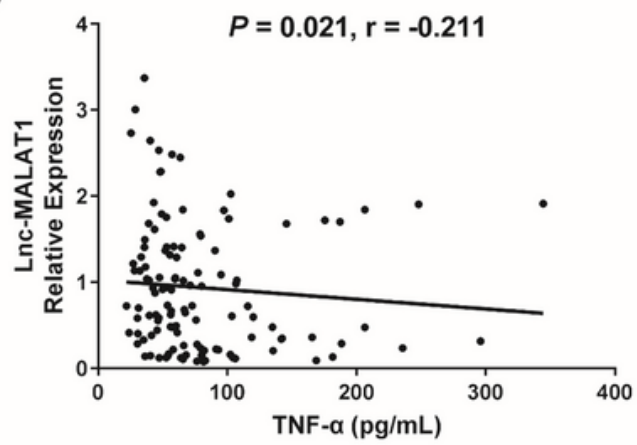

D

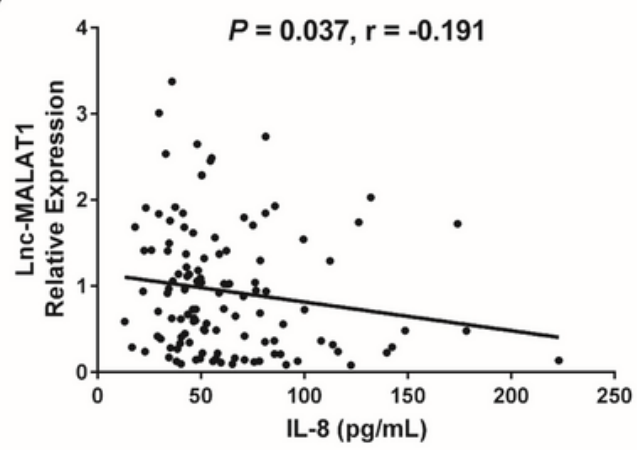

$\mathbf{F}$

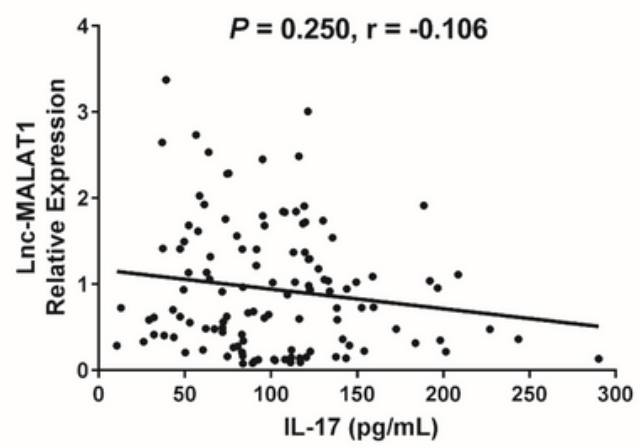

\section{Figure 3}

Correlation of Inc-MALAT1 expression with inflammatory factors levels. Correlation of Inc-MALAT1 expression with CRP (A), TNF-a (B), IL-6 (C), IL-8 (D), IL-10 (E), IL-17 (F), IL-22 (G) was performed. Correlation of Inc-MALAT1 with inflammatory cytokines was determined by Spearman rank test. LncMALAT1, long non-coding RNA metastasis-associated lung adenocarcinoma transcript 1; CRP, C-reactive 
protein; TNF-a, tumor necrosis factor a; IL-6, interleukin-6; IL-8, interleukin-8; IL-10, interleukin-10; IL-17, interleukin-17; IL-22, interleukin-22. $\mathrm{P}<0.05$ was considered significant.

A

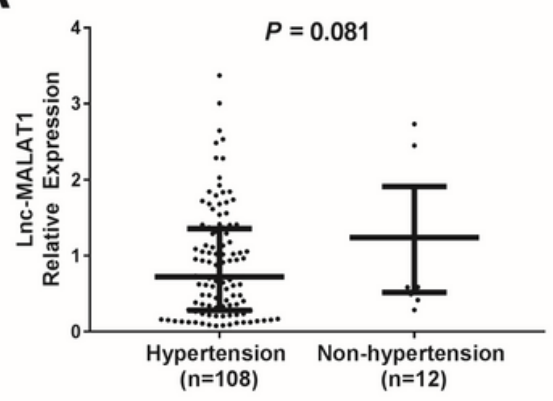

D

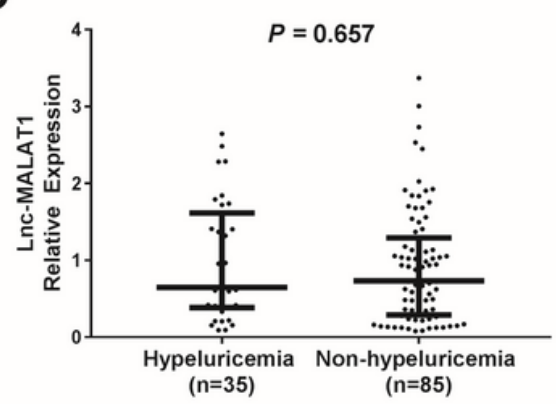

B

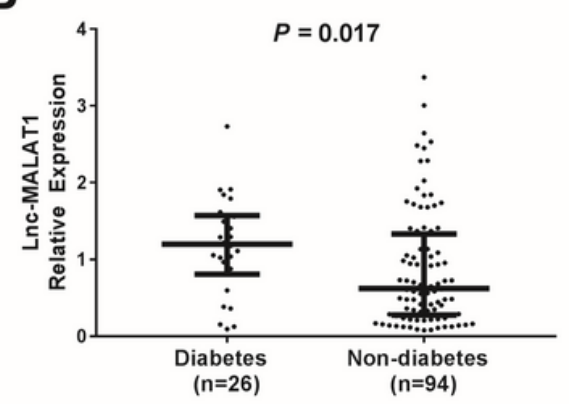

E

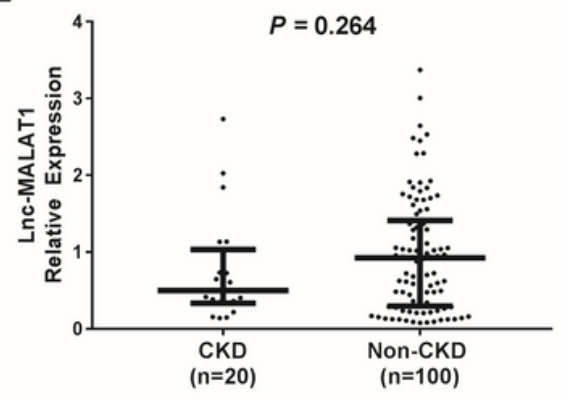

C

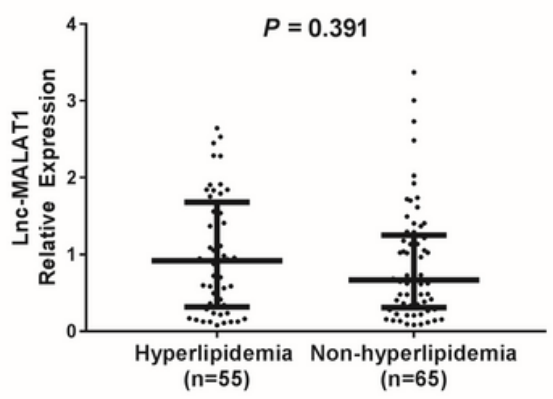

Figure 4

Lnc-MALAT1 expression in AIS patients with different complications. Lnc-MALAT1 in hypertension and non-hypertension patients (A), diabetes patients and non-diabetes patients (B), hyperlipidemia and nonhyperlipidemia patients (C), hypeluricemia and non-hypeluricemia patients (D) as well as CKD and nonCKD patients (E). Comparison between two groups was determined by Wilcoxon rank sum test. LncMALAT1, long non-coding RNA metastasis-associated lung adenocarcinoma transcript 1; AIS, acute ischemic stroke; CKD, chronic kidney disease. $\mathrm{P}<0.05$ was considered significant. 


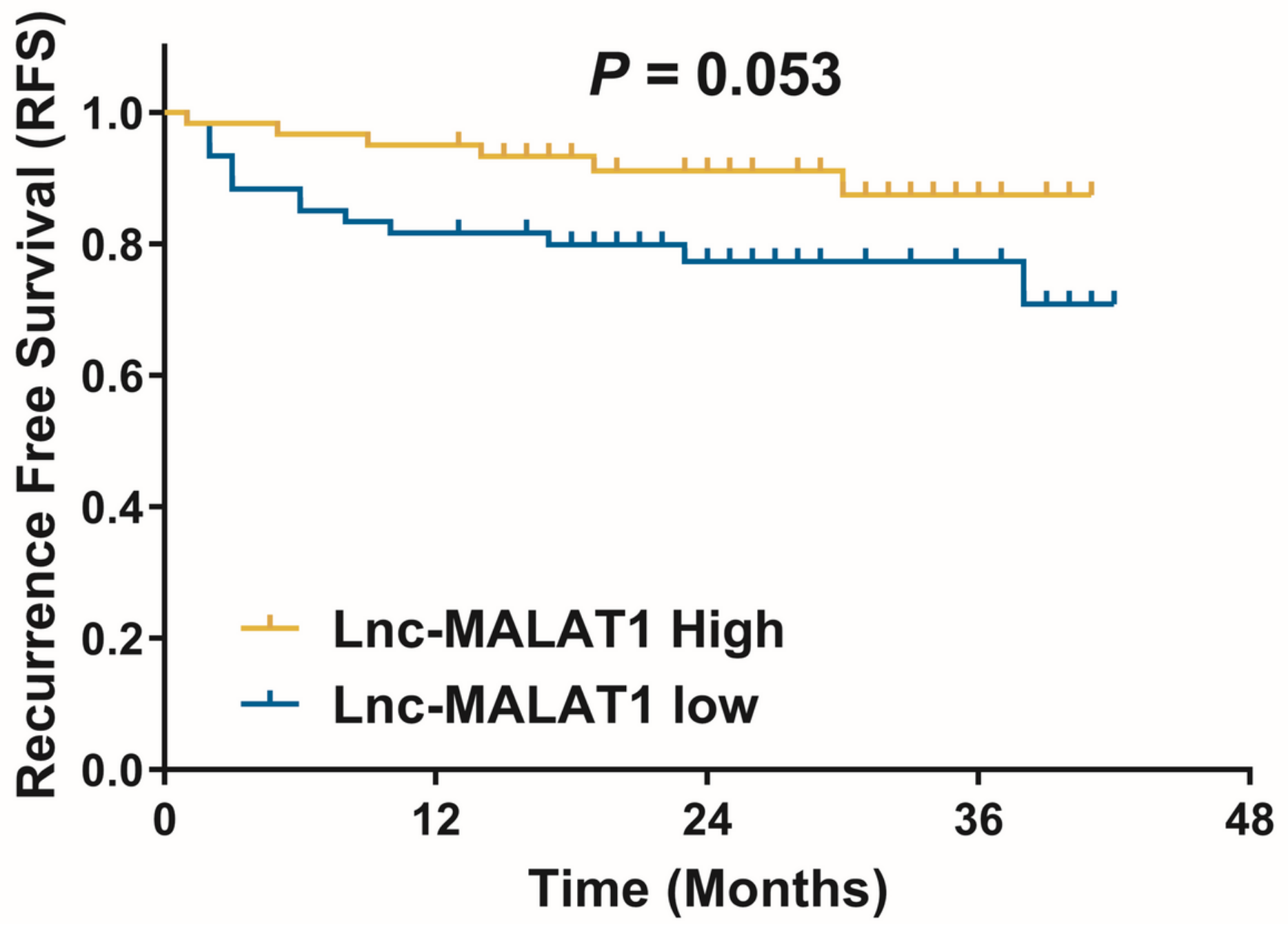

Figure 5

K-M curves analysis. K-M curves displayed the RFS in Inc-MALAT1 high expression and Inc-MALAT1 low expression patients. Comparison of RFS between two groups was determined by log-rank test. RFS, recurrence free survival; Lnc-MALAT1, long non-coding RNA metastasis-associated lung adenocarcinoma transcript 1; K-M curves, Kaplan-Meier curves. $\mathrm{P}<0.05$ was considered significant. 